\title{
Atomic processes in optically thin plasmas
}

\section{Jelle S. Kaastra ${ }^{1,2,3}$, Liyi Gu${ }^{1}$, Junjie Mao ${ }^{1,2}$, Missagh Mehdipour ${ }^{1}$, Ton Raassen ${ }^{1}$ and Igone Urdampilleta ${ }^{1,2}$}

\author{
${ }^{1}$ SRON Netherlands Institute for Space Research, Sorbonnelaan 2, 3584 CA Utrecht, \\ The Netherlands \\ email: j.kaastra@sron.nl \\ ${ }^{2}$ Leiden Observatory, Leiden University, PO Box 9513, 2300 RA Leiden, the Netherlands \\ ${ }^{3}$ Department of Physics and Astronomy, Universiteit Utrecht, P.O. Box 80000, 3508 TA \\ Utrecht, the Netherlands
}

\begin{abstract}
The Universe contains a broad range of plasmas with quite different properties depending on distinct physical processes. In this contribution we give an overview of recent developments in modeling such plasmas with a focus on X-ray emission and absorption. Despite the fact that such plasmas have been investigated already for decades, and that overall there is a good understanding of the basic processes, there are still areas, where improvements have to be made that are important for the analysis of astrophysical plasmas. We present recent work on the update of atomic parameters in the codes that describe the emission from collisional plasmas, where older approximations are being replaced now by more accurate data. Further we discuss the development of models for photo-ionised plasmas in the context of outflows around supermassive black holes and models for charge transfer that are needed for analyzing the data from the upcoming ASTRO-H satellite.
\end{abstract}

Keywords. Atomic data, atomic processes, radiation mechanisms: general, X-rays: general

\section{Introduction}

X-ray emitting plasmas are found everywhere in the Universe, from the small scales of the Solar system up to the large-scale cosmic web filaments. These plasmas show a broad range of environments and physical conditions, for instance collisionally ionised, photo-ionised or transiently ionised. High-resolution X-ray spectroscopy is the key tool to understand these sources. While a lot of progress has been made in the last 15 years using the grating spectrometers on the XMM-Newton and Chandra observatories for point sources, high-resolution X-ray spectroscopy for extended sources has been limited to only the most compact sources on the sky, typically smaller than a few arcmin and using the XMM-Newton RGS detector.

In 2016 Japan will launch ASTRO-H with the SXS (soft X-ray spectrometer) calorimeter, the first detector able to obtain high-resolution spectra of extended sources and with unsurpassed spectral sensitivity in the Fe-K band around $6 \mathrm{keV}$. The older models that are being used to model astrophysical sources are not always up to date with respect to these future capabilities, and therefore efforts are being made to improve the atomic data and models that are being used for X-ray spectroscopy.

There is a strong need to model these different sources with the same, consistent set of atomic parameters. In this contribution we give an outline of recent work in this field done by the authors at SRON. 


\section{Strategy}

The first X-ray spectroscopic models at SRON were developed by Rolf Mewe. With his background as atomic physicist he was hired in 1970 by SRON to model the Solar X-ray spectra obtained by people from this group. His first paper dealt with He-like line ratio's (Mewe 1972a) followed one month later by his first paper with a list of 260 lines in the 1-60 A range (Mewe 1972b). Both papers and follow-up work showed a basic effective strategy to model such spectra, by parameterising the relevant physical processes using simple formulae that are accurate enough relative to the original calculations, and interpolate along isoelectronic sequences, where data are lacking.

The work by Mewe and co-workers gradually evolved and culminated in two papers about continuum emission (Mewe, et al. 1986) and line emission (Mewe, et al. 1985), the latter paper with 2167 lines between $1 \AA$ and $300 \AA$. Some minor corrections, major improvements in the Fe-L complex and addition of lines up to $2000 \AA$ (now totalling 5408 lines) were done later in the mekal model that is implemented in e.g., the XSPEC package.

Starting in 1992, the spectral code was incorporated in a broader scope spectral analysis package, named SPEX (Kaastra, et al. 1996), that allows the user to do spectral data analysis (fitting), plotting, and diagnostic output of physical quantities. In late 2015, version 3 of this package is expected to be released.

Similar developments took place in the USA led by John Raymond, evolving eventually in the APEC model, and in Italy by Landini and Monsignori-Fossi, evolving eventually in the CHIANTI code.

Driven by the requirements of new instrumentation, or by the extension of the present models to more extreme environments, updates of the atomic data are needed.

For example, in collisional ionisation equilibrium plasmas, for most lines collisional excitation by free electrons is the dominant line emission process, and other processes such as radiative recombination contribute only a small fraction of the total line power. For this reason, Mewe had approximated the radiative recombination contribution to the line power as a function of temperature $T$ by a local power-law, proportional to $T^{-0.7}$. This is a reasonable approximation for temperatures close to the temperature, where the relevant ion has its peak concentration. However, radiative recombination rates scale approximately as $T^{-0.5}$ and $T^{-1.5}$ at very low and high temperatures, respectively. Thus, for instance for photo-ionised plasmas, where the temperature is relatively low compared to the typical ionisation potentials, the recombination rates are overestimated using this approach, and a similar situation occurs at high energies for ionising plasmas such as found for example in supernova remnants. Thus, more accurate approximations are needed.

Despite all this evolution of the models, the basic strategy remains unchanged. The code must allow options for fast calculation and yet be accurate enough. This means that the number of mathematical operations and data storage for the basic cross sections or plasma rates need to be minimised. Therefore we follow the same strategy as Mewe used long ago: we use simple, accurate and fast approximations, but more accurate and complete than before.

In addition, since the spectral resolution in the X-ray band in the near future will still be limited as compared for instance to the optical band, we restrict our models to all elements with nuclear charge $Z<30$. Higher elements have too low abundances to give detectable X-ray lines. 
Finally, for a full model, we need updates for many processes, such as the collisional and photo-ionisation cross sections, radiative transition probabilities, auto-ionisation rates, recombination rates, line energies, etc.

Since this is a process, where it easy to make mistakes, and sometimes small changes in specific parameters can have a large effect on parts of the spectrum, a careful comparison of our results with both the older calculations and other plasma models will be made.

\section{Examples}

\subsection{Collisional ionisation rates}

In the past, several compilations of direct collisional ionisation rates have been made (here collisions with free electrons are assumed). One of the most recent compilations was the one given by Dere (2007). He has given a thorough review of cross sections and has calculated total ionisation rates for each ion in a plasma, listing it at typically 20 spline points on a scaled temperature grid.

Since the review by Dere newer data have become available. But more importantly, since Dere lists total rates, the individual contributions of the atomic subshells are not available, and these are needed for instance in order to calculate the fluorescence contribution to spectral lines after inner-shell ionisation. We have therefore started a project of updating the database of Dere, but obtaining parameterised forms of the cross-sections for individual subshells. These parameterised forms are chosen in such a way that they can be integrated analytically over a thermal Maxwell-distribution or other simple forms of electron distributions. This work will be reported by Urdampilleta et al. (in prep.).

\subsection{Radiative recombination}

For radiative recombination, extensive material exists that gives the total recombination rates, added over all possible higher shells, into which the recombining electron is captured. Also in this case we need rates to individual levels in order to be able to calculate the corresponding emission line spectrum. In addition, not only the capture rates to individual levels are of interest, but also the associated cooling rates, the kinetic energy of the captured electron averaged over the recombination rate. This quantity is of importance for photo-ionised plasmas.

We have started collecting data from the literature or made our own calculations, and parameterise the plasma rates by a relatively simple seven-parameter equation that gives an accuracy of $<5 \%$ for most recombinations. This work will be presented by Mao et al. (in prep.).

\subsection{Photo-ionised plasmas}

In recent work on photo-ionised plasmas, it appeared to us that in some cases there are apparently large differences between the calculated photo-ionisation equilibria using different spectral models like Cloudy, XSTAR, and our own pion model in the SPEX package. Photo-ionised plasmas are the most challenging environments, because of the large number of physical processes that play a role. We have started comparing the individual processes that are used in these models in detail. For example, it appears that in some work the radiative recombination approximation of Seaton (1959) for hydrogen is used, which gives an accurate approximation up to $k T$ of about $300 \mathrm{eV}$, but overestimates the recombination rate significantly at higher energies. As a consequence, the cooling due to radiative recombination is overestimated by more than an order of magnitude for temperatures of a few $\mathrm{keV}$ or higher. Other important differences concern the treatment 
of Compton scattering in the heating or cooling balance. A systematic comparison will be presented elsewhere (Mehdipour et al., in prep.).

\subsection{Helium-like line ratios in active galactic nuclei}

The so-called $R$-ratio between the forbidden and intercombination line in helium-like ions is known to be a good density indicator. At low densities, $R$ is high, and then it quickly drops above a critical density that depends on the ion that is considered. However, in some AGN $R$-ratios have been found that are even higher than the low-density limit, and therefore cannot be explained. But because the intercombination line of the He-like triplet is close in energy to energy of the $1 \mathrm{~s}^{2} 2 \mathrm{~s}$ to $1 \mathrm{~s} 2 \mathrm{~s}\left({ }^{1} \mathrm{~S}\right) 2 \mathrm{p}{ }^{2} \mathrm{P}$ doublet in Li-like ions, and for sufficiently large Doppler broadening and column density of the Li-like ion, the Li-like ions can absorb a significant fraction of the intercombination line flux, thereby artificially increasing the apparent $R$-ratio (Mehdipour, et al. 2015). Thus, depending on the precise physical conditions, care must be taken in interpreting $R$-ratios.

\subsection{Charge transfer modelling}

Charge transfer is the process, where an ion collides with another ion, neutral atom or molecule, and where the ion either obtains an electron from the other particle, or looses an electron. Charge transfer recombination of ions with neutral hydrogen atoms is an important process in many situations, in particular at the interface of cold and hot material, like comets, the solar wind, supernova remnant shocks, etc. We have started a project ( $\mathrm{Gu}$ et al., in prep.), where we collect all available cross section data for this process from the literature and databases, derive from them scaling laws for the distribution over $n$ and $\ell$, dependent of the velocity difference between both particles, and apply these scaling laws to the other elements and ions, for which no direct data exists. Radiative cascade calculations are taken into account up to $n=16, \ell=15$. Preliminary tests of this $c x$ model in SPEX show that it gives an excellent fit to the X-ray spectrum of comet C2000 WM1.

\section{Conclusions}

Astrophysical sources are sometimes found in remarkable areas of parameter space. New X-ray missions like ASTRO-H (launch 2016) demand more details and accuracy. We showed examples of the work in progress at SRON: updates of the atomic parameters in our X-ray spectral models to account for this. SPEX (www.sron.nl/spex) Version 3 will contain these updates (release late 2015).

\section{References}

Dere, K. P. 2007, A\&\&A, 466, 771

Kaastra, J. S., Mewe, R., \& Nieuwenhuijzen, H. 1996, in: 11th Colloquium on UV and X-ray Spectroscopy of Astrophysical and Laboratory Plasmas, p. 411

Mehdipour, M., Kaastra, J. S., \& Raassen, A. J. J. 2015, A\& $A$, 579, A87

Mewe, R. 1972a, Solar Physics, 22, 114

Mewe, R. 1972b, Solar Physics, 22, 459

Mewe, R., Gronenschild, E. H. B.. M., \& van den Oord, G. H. J.. 1985, A\&GAS, 62, 197

Mewe, R., Lemen, J. R., \& van den Oord, G. H. J.. 1986, A\&ऽAS, 65, 511

Seaton, M. J. 1959, MNRAS, 119, 81 